\title{
Metabolomics Reveals the Effects of Copper on Mitochondria-mediated Apoptosis in Kidney of Chicken (Gallus Gallus)
}

Jianzhao Liao

South China Agricultural University

Fan Yang

Jiangxi Agricultural University

Yuman Bai

South China Agricultural University

Wenlan Yu

South China Agricultural University

$\mathrm{Na}$ Qiao

South China Agricultural University

Qingyue Han

South China Agricultural University

Hui Zhang

South China Agricultural University

Jianying Guo

South China Agricultural University

Lianmei Hu

South China Agricultural University

Ying Li

South China Agricultural University

Jiaqiang Pan

South China Agricultural University

Zhaoxin Tang ( $\square$ tangzx@scau.edu.cn )

South China Agricultural University

\section{Research}

Keywords: copper, metabolomics, mitochondrion, apoptosis, kidney

Posted Date: March 26th, 2021

DOI: https://doi.org/10.21203/rs.3.rs-361388/v1 
License: (c) (i) This work is licensed under a Creative Commons Attribution 4.0 International License. Read Full License 


\section{Abstract}

Background: Copper (Cu), a common feed additive in diets for animals, is effective in improving growth performance and feed efficiency. However, excessive intake of $\mathrm{Cu}$ can cause toxic effects. Kidney is the main target organ of $\mathrm{Cu}$, but the relationship between $\mathrm{Cu}$-induced nephrotoxicity and its metabolic process remains unclear.

Results: For deeply investigating the nephrotoxicity induced by $\mathrm{Cu}$, a total of 240 broiler chicks were fed with different contents of $\mathrm{Cu}(11,110,220$, and $330 \mathrm{mg} / \mathrm{kg} \mathrm{Cu}$ ) for $49 \mathrm{~d}$ in this study (60 chicks per group). The results of TUNEL staining showed that $\mathrm{Cu}$ could induce apoptosis in kidney with increasing of TUNEL-positive cells. Additionally, a total of 62 differential metabolites were detected by liquid chromatography-mass spectrometry (LC-MS), and mainly enriched in the metabolic pathways including riboflavin metabolism, glutathione metabolism, sphingolipid metabolism, and glycerophospholipid metabolism, which were closely related to mitochondrial metabolism. Meanwhile, the decrease of mitochondrial membrane potential, impairment of mitochondrial respiratory function, and the increase of mitochondrial membrane permeability indicated that renal mitochondria were damaged by excess $\mathrm{Cu}$. Furthermore, the increase of mRNA and protein levels of Drp1, Bax, Bak-1, CytC, Caspase-3/cleaved Caspase-3 and the decrease of mRNA and protein levels of OPA1, Mfn1, Mfn2, and Bcl-2 confirmed that $\mathrm{Cu}$ could induce mitochondria-mediated apoptosis in kidney.

Conclusions: These results highlighted that mitochondrial metabolism could be considered as an important factor in influencing Cu toxicity, which for further demonstrating that Cu could induce mitochondria-mediated apoptosis in kidney of broilers.

\section{Background}

As a necessary trace element for poultry, copper (Cu) plays a positive effect in mitochondrial respiratory, hematopoiesis, and anti-oxidative function in organisms [1, 2]. However, excess $\mathrm{Cu}$ can lead to toxicity by disturbing osmotic regulation and causing metabolic disturbance [3, 4, 5]. Due to the positive and negative effects of $\mathrm{Cu}$ on animal health, the content of $\mathrm{Cu}$ in feed has been raised concern. In practice, adding $\mathrm{Cu}$ to feedstuffs for poultry can improve growth performance and feed efficiency. Nevertheless, long-term excessive intake of $\mathrm{Cu}$ can cause toxic effects in animals and humans due to the enrichment of the food chain. Especially for mitochondria, excess Cu can disrupt redox balance by impairing the respiratory enzyme complexes, which leads to reactive oxygen species (ROS) formation, catalyzes Fenton/Haber-Weiss reactions and thus makes damaged to antioxidant defense system $[6,7,8]$. Previous studies have demonstrated that large amounts of $\mathrm{Cu}$ were found in various animals, including poultry [9, 10]. Additionally, the renal toxicity induced by $\mathrm{Cu}$ has been widely concerned since the kidney is the target organ of $\mathrm{Cu}$ [11]. Indeed, numbers of studies pointed out that $\mathrm{Cu}$ could cause nephrotoxicity via reactive oxygen production, energy deficiency, and mitochondrial dysfunction $[11,12]$. 
It is universally acknowledged that oxidative damage and mitochondrial toxicity are associated with apoptosis. Apoptosis, an active and gene-control cell death, is highly regulated within metabolic process. It is characterized by chromatin condensation, DNA cleavage, and the formation of apoptotic bodies [13]. The mechanism of apoptosis is mostly complex and mainly divided to endogenous and exogenous pathways. The endogenous pathway, known as mitochondria-mediated pathway, can be activated whereby stimuli in the extracellular matrix, which induce permeation of the outer mitochondrial membrane (OMM) and collapse of mitochondrial membrane potential [14]. In this process, numbers of signal molecules such as Bax (pro-apoptotic) or Bcl-2(anti-apoptotic) can be recruited to OMM, which result in the decrease of the mitochondrial membrane potential (MMP), and cytochrome $C$ (CytC) was released to the cytoplasm. CytC can promote the formation of apoptosome as a death factor, which activates Caspase-3 by stimulating enzymatic reactions and leads to apoptosis [15]. At present, the nephrotoxicity of Cu has attracted widespread attention, and apoptosis is considered as one of the underlying mechanisms of Cu poisoning.

It is reported that mitochondrial morphology is closed related to apoptosis [16]. Actually, the maintenance of mitochondrial morphology depends on its fusion and fission in normal physiological state. The dynamic balance in mitochondria is crucial for maintaining cellular homeostasis. When mitochondrial dynamics is in imbalance, the metabolic processes such as energy generation, immunity, and apoptosis will be affected [17]. Recent studies have pointed out that numbers of components related to fission and fusion machinery implicated in mitochondrial shape changes including dynamin-related protein 1 (Drp1) and mitochondrial fusion proteins (Mfn1, Mfn2, and OPA1) are indispensable for apoptosis [18, 19]. In addition, some of findings revealed that excessive mitochondrial fission could trigger the increasing open degree of mitochondria permeability transition pore (MPTP) and promote the release of CytC from mitochondria, leading to mitochondrial-mediated apoptosis [18, 20].

Cellular metabolism and the pathways of cell death are regarded as the important factors in regulating cell fate. When the cell homeostasis is disrupted, the metabolites should be changed due to cell function affected. If metabolic energy is not enough to satisfy the requirements of biosynthesis and homeostasis maintenance, cells will cease to function normally and trend to apoptosis eventually. Thus, the detection of metabolite change has certain significance for reflecting the physiological state of the body. In recent years, metabolomics techniques are widely used in toxicology and disease diagnosis. Metabolomics is the method for quantitative analysis of the overall variation of metabolic substances in a certain period, which can firsthand reflect the final status of biosystems, and it can reveal the relationship between metabolites and physiological or pathological changes [21]. Various studies have showed that the metabolites play a key role in phenotypic regulation and biological synthesis by interacting with genes and proteins $[22,23]$. Indeed, the changes of intracellular metabolites may reveal the metabolic pathway during the process of apoptosis [24]. In present research, liquid chromatography-mass spectrometry (LCMS) was used to detect the changes in metabolites in kidney, and mitochondria dynamics and apoptosis was explored, which further explore the role of mitochondria-mediated apoptosis on Cu-induced nephrotoxicity from the point of metabolomics. 


\section{Methods And Materials}

\section{Animals and treatment}

A total of 240 newborn chicks ( 1 day of age, weighting $43 \pm 0.32 \mathrm{~g}$ ) were divided into 4 groups at random (including one control group and three treated groups, 60 broiler chicks per group, half male and half female). The ingredient and nutritional levels of the basic feed were followed in Table S1, and Cu sulfate was selected as $\mathrm{Cu}$ sources. Broilers were fed with the basal ration containing $11 \mathrm{mg} \cdot \mathrm{kg}^{-1} \mathrm{Cu}$ in control group, and the Cu levels in three treated groups were as follow: $110 \mathrm{mg} \cdot \mathrm{kg}^{-1} \mathrm{Cu}, 220 \mathrm{mg} \cdot \mathrm{kg}^{-1} \mathrm{Cu}$, and $330 \mathrm{mg} \cdot \mathrm{kg}^{-1} \mathrm{Cu}$, respectively, which was referred to the previous studies $[3,6,25]$. All the broilers were given ad libitum access to water and diet. On the day 49 of the treatment, the serum was collected for blood biochemistry test, and the kidneys were collected from broilers after euthanasia with nembutal. The procedures were authorized by the Ethics Committee of South China Agricultural University (Permission number: NO. 2017A087).

\section{Histological observation}

Tissues were fixed in $4 \%$ paraformaldehyde for $24 \mathrm{~h}$. The steps of making pathological section and hematoxylin eosin (H\&E) staining were referred to our previous studies $[3,6]$. The renal tubular damage score was obtained according to the previous studies [26, 27].

\section{TUNEL staining assay}

Kidney tissues were immobilized in $4 \%$ paraformaldehyde and paraffin-embedded tissue slices were prepared referring to the standard procedure. TUNEL assay was executed by TUNEL test kit (Beyotime, China) based on the guidance note. Sections were treated with proteinase $\mathrm{K}$ and hydrogen peroxide. Then, the sections were incubated with biotin-dUTP solution at $37^{\circ} \mathrm{C}$ at dark for $60 \mathrm{~min}$. After being washed with phosphate buffer solution (PBS), the Streptavidin-HRP detected solution was used to incubate $30 \mathrm{~min}$ at room temperature and then incubated with diaminobenzidine (DAB). Hematoxylin was used for nuclear labeling. The positive rate of TUNEL staining was measured by Image J.

\section{Ultrastructural observation}

The preparation of ultrathin sections and the acquisition of electron microscope photos were performed by the Test Center of South China Agricultural University. The specific methods could be found in supplementary materials.

\section{ROS detection}

ROS was detected by fluorescent probe DCFH-DA by flow cytometry according to the guidance note (Beyotime, China). Briefly, the kidney tissues were digested into individual cells and DCFH-DA was used to incubate for $20 \mathrm{~min}$ at $37^{\circ} \mathrm{C}$. Fluorescence intensity was analyzed by flow cytometry.

\section{Metabolomics analysis}


$50 \mathrm{mg}$ of tissues in control group and $330 \mathrm{mg} / \mathrm{kg}$ Cu group were taken for metabolite extraction. The method of sample extraction and the analytical equipment platform for LC-MS was from Waters, and the parameter setting was also referred to Luan et al. [28]. The original data were processing by employing for baseline filtration, peak extraction, integration, time correction, peak calibration, and normalization. The resultant data were processed into SIMCA-14.1 software (Umetrics, Sweden) and imported to principle component analysis (PCA) and orthogonal partial least squared discriminant (OPLS-DA). The variable importance in the projection (VIP) $>1$ and $P<0.05$ were identified as the potential metabolic markers. The metabolites were identified by comparing with HMDB online databases and the metabolic pathways were analyzed by KEGG pathway database and MetaboAnalyst 4.0.

\section{Measurement of mitochondrial oxygen consumption}

The extraction of renal mitochondria was performed referring to previous study [29]. The mitochondrial oxygen consumption was detected by Oxygraph-2k instrument ( $02 k$, Oroboros instruments, Austria) at 37 $\pm 0.001^{\circ} \mathrm{C}$. After protein quantification, the same amounts of mitochondria were added to the respiration chamber containing $2 \mathrm{~mL}$ of respiration medium (The recipe was seen in supplementary materials). When the oxygen consumption rate $(\mathrm{OCR})$ tended to balance, succinate, pyruvate, malate, adenosine diphosphate (ADP), carbonyl cyanide m-chlorophenylhydrazone (CCCP), rotenone, and antimycin A were sequentially added. OCR was calculated using the DatLab 4 analysis software. The routine respiration, respiratory control ratio (RCR), maximal respiration (MAX), ATP-linked respiration, and Spare respiratory capacity (SRC) were calculated by the description in Fig. S1 [29, 30].

\section{Determination of MMP and MPTP}

MMP was detected by JC-1 assay (Thermo Fisher Scientific, USA) following the guidance note. Briefly, the kidney tissues were digested into individual cells and washed with PBS for three times. JC-1 was used to incubate for $20 \mathrm{~min}$ at $37^{\circ} \mathrm{C}$. After washing, cells were analyzed under the emission wavelength of 590 $\mathrm{nm}$ and $525 \mathrm{~nm}$ by flow cytometry.

MPTP was determined by MPTP Assay Kit with Calcein AM fluorescence probe based on the guidance note (Beyotime, China). Briefly, the kidney tissues were digested into individual cells and washed in PBS for three times. Cells were incubated with Calcein AM staining solution in a dark room for 30 min at $37^{\circ} \mathrm{C}$. Then, Calcein AM staining solution was removed and the cells were resuspended in DMEM at $37^{\circ} \mathrm{C}$ for 30 min in the dark. After washing, fluorescence intensity was analyzed by flow cytometry.

\section{mRNA expression analysis}

Total RNAs were extracted and reverse transcribed to cDNA referring to previous study [8]. Gene-specific primers of Drp1, OPA1, Mfn1, Mfn2, Bax, Bak-1, Bcl-2, p53, CytC, Caspase-3, and GAPDH were shown in Table S2. RT-qPCR was performed on QuantStudio 3 Real-Time PCR System (ThermoFisher, USA). GAPDH was used as the reference gene and the mRNA expression was calculated according to $2^{-} \triangle \triangle \mathrm{CT}$ method.

\section{Western blot analysis}


The total proteins were extracted with radioimmunoprecipitation assay (RIPA) lysis buffer (Beyotime, China). Then, the extracted protein contents were determined by BCA assay (Beyotime, China). $10 \mathrm{mg}$ of protein was separated by electrophoresis, transferred to membranes, and blocked. Incubation of primary and secondary antibodies was proceeded as previously described [3]. After washing with TBST, ECL solution (Millipore, U.S.A.) was used to visualize the blot. The protein expression was analyzed by Image J.

\section{Statistical analysis}

The experimental data were analyzed using the SPSS 22.0 (SPSS Inc., U.S.A.) and GraphPad Prism 8.0.1 (GraphPad Inc., U.S.A.). Results were presented as mean \pm S.E.M. $(n=6$, repeated thrice), and the $P$ value $<0.05$ was considered significant.

\section{Results}

\section{Evaluation of renal injury induced by $\mathrm{Cu}$}

In this study, the body weight of broilers was significantly increased in $220 \mathrm{mg} / \mathrm{kg}$ Cu group and remarkably decreased in $330 \mathrm{mg} / \mathrm{kg}$ Cu compared to control group (Fig. S2). Furthermore, the Cu content in serum and kidney and the biochemical indicators (BUN, Crea, and UA) were increased with increased intake of $\mathrm{Cu}$ (Table S3-4). The kidney coefficient was obtained to analysis the pathological effects induced by $\mathrm{Cu}$. As presented in Fig. 1D, the kidney coefficient was elevated after treating $220 \mathrm{mg} / \mathrm{kg}$ and $330 \mathrm{mg} / \mathrm{kg} \mathrm{Cu}$ in comparison with control group, which might indicate that pathological swelling could be induced by $\mathrm{Cu}$. In order to verify the pathological damage in kidney induced by $\mathrm{Cu}, \mathrm{H} \& \mathrm{E}$ staining was executed to observe the changes of histological morphology. As shown in Fig. 1A, the vacuoles degenerations in renal tubular epithelial cells could be found in all treated group in comparison with the control group, especially in the $330 \mathrm{mg} / \mathrm{kg}$ Cu group (white arrows). Additionally, renal tubular injury was evaluated with the scores of renal tubular damaged, which showed that the score of tubular injury was significantly increased with elevated $\mathrm{Cu}(P<0.01$ or $P<0.001)$ (Fig. 1E). For exploring whether ROS were the source of kidney injury, ROS levels were evaluated and the results suggested that the ROS production was increased remarkably with the elevated $\mathrm{Cu}(P<0.01$ or $P<0.001)$ (Fig. 1C\&G).

TUNEL staining is the classical method to detect apoptotic cells with DNA fragmentations, and the apoptotic cells were stained with brown. As demonstrated in Fig. 1B, the brown-strained cells (black arrows) in kidney were elevated remarkably with the increase of $\mathrm{Cu}$ level, the color depth and areas were also increased. Quantitative analysis of TUNEL-positive cells also showed the elevated positive rate with the increased $\mathrm{Cu}(P<0.001)$ (Fig. 1F).

\section{Effects of Cu on the change of metabolites in kidney}

In order to investigate the relevancy between metabolic process and apoptosis, the kidney metabolites in the $330 \mathrm{mg} / \mathrm{kg} \mathrm{Cu}$ group and control group were analyzed by metabolomics. The non-targeted metabolomics data were collected in the modes of positive and negative ion by LC-MS. PCA and OPLS- 
DA were used to pattern discriminant analysis. The clustering of QC plots in PCA indicates that the analytical method was high repeatability and low variability (Fig. 2A-B). Additionally, the plots representing the $330 \mathrm{mg} / \mathrm{kg}$ Cu group were significantly separated from those of control group in both positive and negative modes, which indicated a significant change of the metabolites and a success of modeling (Fig. 2).

The differential metabolic substances were chosen on the basis of VIP $>1, P<0.05$. There were 62 differential metabolites were shown in Fig. 3A\&B (heatmap and volcano plot) and Table. S5\&6 (including positive and negative ion). Among of above, 30 metabolites were elevated and 32 metabolites were reduced in the $330 \mathrm{mg} / \mathrm{kg}$ Cu group in comparison with control group.

\section{The change of metabolic pathways caused by Cu in kidney}

As shown in Fig. 3C, the pathway analysis showed that Cu-induced apoptosis involved 17 metabolic pathways, and the riboflavin metabolism, glutathione metabolism, sphingolipid metabolism, and glycerophospholipid metabolism had the greatest impact. Among the metabolites involved in these metabolic pathways, flavin mononucleotide, phosphatidylethanolamine (44:10), ceramide (d18:1/24:1(15Z)), and 3'-0-sulfogalactosylceramide were increased in $330 \mathrm{mg} / \mathrm{kg}$ Cu group in comparison with control group. Moreover, oxidized glutathione, glutathione, phosphatidylcholine (14:0/24:1), lysophosphatidylcholine (22:5) were reduced in $330 \mathrm{mg} / \mathrm{kg}$ Cu group in comparison with control group (Fig. 3D). The metabolic diagram was shown in Fig. 3E.

\section{$\mathrm{Cu}$ induced mitochondrial damage in kidney}

In this study, mitochondrial morphology was observed by TEM. As shown in Fig. 4A, the nuclei were normal without shrinkage in control group, and mitochondrial membrane structures were intact. Similarly, no obvious pathological changes were observed in the $110 \mathrm{mg} / \mathrm{kg}$ Cu group. However, the nuclear deformation and shrinkage was found in the $220 \mathrm{mg} / \mathrm{kg}$ Cu group, and mitochondrial cristae partly disintegrated (red arrows). What's notable was that mitochondrial vacuolar degeneration, break and disappearance of the mitochondrial cristae could be seen in the $330 \mathrm{mg} / \mathrm{kg}$ Cu group.

Next, the effect of $\mathrm{Cu}$ on mitochondrial respiratory function is measured by real-time OCR using Oxygraph-2k instrument. Schematic diagram was shown in Fig. S1. The respiration without adding any reagents is routine respiration. State 4 is the respiration in presence of specific substrates. State 3 is the respiration with saturated substrate and ADP. RCR was obtained from the rate between state 3 and state 4 respiration. After the addition of oligomycin, the oxygen consumption was decreased, which represented the oxygen consumption exploited for ATP production and known as ATP-linked respiration. Subsequently, mitochondrial uncoupler CCCP was added to maximize mitochondria respiratory for obtaining the Max. Furthermore, SRC could be calculated by the difference between MAX and State 4 respiration. As presented in Fig. 4B, the routine respiration, RCR, MAX, and ATP-linked respiration were decreased remarkably with the increasing Cu level $(P<0.05, P<0.01$ or $P<0.001)$. SRC was showed a down trend with the increase $\mathrm{Cu}$ level, but the difference was not significant $(P>0.05)$. 
Flow cytometry was used to detect the MMP and MPTP, and the results were shown in Fig. 4C-D. JC-1 is widely used as a fluorescent probe to detect MMP. JC-1 exists as monomers at low MMP and emits green fluorescence after laser excitation. However, JC-1 can emit red fluorescence in the form of aggregates at high MMP. Therefore, the transition from red to green fluorescence can reflect a decrease of MMP. As shown in Fig. 4C, the decreased trend of MMP could be detected via the transformation from red to green fluorescence, and the proportion of cells with low MMP were significantly increased with the raising $\mathrm{Cu}$ level, which showed dosage effect $(P<0.001)$. Additionally, the effects of Cu on MPTP in kidney were presented in Fig. 4D. The opening degree of mitochondria membrane pore was inversely proportional to the fluorescence density of Calcein AM. The results showed that the mean fluorescence density of Calcein AM was decreased with the elevated $\mathrm{Cu}$, and the remarkable change was shown in $330 \mathrm{mg} / \mathrm{kg} \mathrm{Cu}$ group in comparison with control $(P<0.01)$.

\section{$\mathrm{Cu}$ induced mitochondrial dynamics disorder in kidney}

As exhibited in Fig. 5A, the mRNA level of Drp1 was enhanced dramatically when treated with $220 \mathrm{mg} / \mathrm{kg}$ and $330 \mathrm{mg} / \mathrm{kg}$ Cu compared with control group $(P<0.01$ or $P<0.001)$. However, the mRNA expression levels of OPA1, Mfn1 and Mfn2 were decreased with the elevated Cu level. The expressions mitochondrial dynamic-related proteins were shown in Fig. 5B and the quantitative result were exhibited in Fig. 5C\&D. The protein expression levels were showed the similar trend of mRNA expression. As shown, the protein expression level of Drp1 up-regulated and OPA, Mfn1, and Mfn2 down-regulated with the elevated Cu level.

\subsection{Cu induced mitochondria-mediated apoptosis in kidney}

Mitochondria-mediated apoptosis is controlled by multitudinous signal factors. Thus, the mRNA and protein expression of Bak-1, Bax, Bcl-2, CytC, cleaved Caspase-3 and the mRNA expression of p53 were detected and the results were seen in Fig. 6. The mRNA and protein expression levels of Bak-1, Bax, CytC, and cleaved Caspase-3 were enhanced with the elevated $\mathrm{Cu}$, and the remarkable difference was exhibited in $220 \mathrm{mg} / \mathrm{kg} \mathrm{Cu}$ and $330 \mathrm{mg} / \mathrm{kg}$ Cu group in comparison with control group $(P<0.05, P<0.01$ or $P<$ $0.001)$. Additionally, the mRNA expression of p53 in all treated groups were significantly enhanced compared to control group $(P<0.05, P<0.01$ or $P<0.001)$. Unlike the others, the mRNA and protein expression levels of $\mathrm{Bcl}-2$ were memorably reduced in $330 \mathrm{mg} / \mathrm{kg}$ Cu compared with control group $(P<$ 0.05).

\section{Discussion}

$\mathrm{Cu}$ is an essential nutrient in poultry, which is usually prescribed to increase feed efficiency and improve growth performance. However, Cu can be toxic to poultry when ingested overdose. Kidney is considered as the storage organ for $\mathrm{Cu}$, so it is of important significance to explore the nephrotoxicity induced by $\mathrm{Cu}$. Previous study had found that Cu could accumulate in kidney and cause pathological damage [11], and the elevated ROS levels and injury scores also confirmed the renal damage could be caused by $\mathrm{Cu}$ in present study. In addition, many studies indicated that $\mathrm{Cu}$ could induce apoptosis, but the metabolic 
changes and metabolic processes involved in Cu-induced apoptosis were poor studied. In this study, metabolomic techniques were used to detect Cu-induced metabolite differences and metabolic pathways, which may reflect the effects of mitochondria-dependent apoptosis in kidney induced by $\mathrm{Cu}$.

In this study, TUNEL staining assay was used to detect Cu-induced apoptotic cells in kidney. The obtained results of TUNEL staining showed that the apoptotic cells were significantly increased with the elevated $\mathrm{Cu}$ level. Meanwhile, TEM also showed that the karyopyknosis was found in kidney with the increased intake level of $\mathrm{Cu}$. These results confirmed that apoptosis can be induced by $\mathrm{Cu}$ in kidney, and the doseeffect was shown. Therefore, the samples in the control group and the $330 \mathrm{mg} / \mathrm{kg}$ Cu group were selected for metabolomics analysis to reveal the metabolic pathway of Cu-induced apoptosis in the kidney.

The results of metabolomics showed that a total of 62 differential metabolites were associated with Cuinduced nephrotoxicity in kidney, which mainly affected riboflavin metabolism, glutathione metabolism, sphingolipid metabolism, and glycerophospholipid metabolism. Riboflavin metabolism is closely linked to mitochondrial energy metabolism. In this metabolic pathway, flavin adenine dinucleotide and flavin mononucleotide are the two important redox cofactors in mitochondrial respiratory chain, which are vital for energy production. Some studies pointed out that flavoprotein might be involved in superoxide production, which revealed that riboflavin metabolism might play a part in apoptosis via oxidative stress $[31,32]$. Additionally, glutathione metabolism is an essential metabolic pathway for cellular growth and homeostasis. It is reported that the low level of glutathione can lead to high level of mitochondrial ROS and cause the oxidative damage to proteins, lipids, and DNA [33]. Moreover, sphingolipid metabolism, a complex network composed of interconnected metabolites with ceramide as the central hub, which can cooperate with Bax and Bak to increase mitochondrial metabolic efficiency. The role of sphingolipid metabolism during apoptosis has been extensively explored [34, 35]. As the hub in sphingolipid metabolism, the increased level of ceramide can respond to the pro-apoptotic signal, inducing the key factors in the apoptosis cascade. Meanwhile, mitochondrial fusion/fission and apoptosis are always accompanied by changes in the glycerophospholipid composition (including phosphatidylethanolamine, phosphatidylcholine, lysophosphatidylcholine and so on) on the out surface of cell membranes or organelles membranes, which is extremely related to glycerophospholipid metabolism pathway [36]. In this study, $330 \mathrm{mg} / \mathrm{kg}$ Cu could increase the level of flavin mononucleotide to change the level of riboflavin metabolism, which might affect glycerophospholipid metabolism pathway (decreased levels of phosphatidylcholine, lysophosphatidylcholine and increased level of phosphatidylethanolamine) and glutathione metabolism pathway (decreased levels of oxidized glutathione and glutathione) via pentose and glucuronate interconversion and TCA cycle. Additionally, Cu-induced significantly increased levels of ceramide (d18:1/24:1(15Z)) and 3'-0-sulfogalactosylceramide resulted in the change of sphingolipid metabolism pathway also be related to the change of glycerophospholipid metabolism and glutathione metabolism. The metabolomics results suggested that the abnormal activities of riboflavin metabolism, glutathione metabolism, sphingolipid metabolism, and glycerophospholipid metabolism were probably related to Cu-induced apoptosis in kidney. 
Interestingly, the differences metabolites and metabolic pathways caused by $\mathrm{Cu}$ exposure in present study were closely related to mitochondrial dysfunction. Thus, the mitochondria-mediated apoptosis induced by $\mathrm{Cu}$ in kidney was investigated. Firstly, mitochondrial morphology and function were evaluated. The results of TEM showed mitochondrial cristae fracture and vacuolization in Cu treatment groups. Besides that, the respiratory function detection of the extracted mitochondria also reflected the toxic effect of $\mathrm{Cu}$ on renal mitochondria. In this study, the OCR was increased immediately after adding substrates, which confirmed that extracted mitochondria were active. Among the relevant indicators obtained, RCR can well reflect the mitochondrial function and status. A high level of RCR suggest the high coupled effect between ATP production and electron transport in respiratory chain. Meanwhile, ATP-linked respiration represents the oxygen consumption linked to ADP phosphorylation. MAX is depended on the activity of the complex enzymes. Additionally, the SRC is considered as the capacity of the mitochondria to respond to the energy requirement, which is a measure of cell's fitness [30]. Our study found that the routine respiration, RCR, MAX, and ATP-linked respiration were decreased significantly with the increasing level of $\mathrm{Cu}$, and SRC was also shown a down trend. These indicated that $\mathrm{Cu}$ could weaken the ATP synthesis, electron transport efficiency, and energy storage capacity in renal mitochondria. Moreover, the results of flow cytometry showed that $\mathrm{Cu}$ could lead to the increase of mitochondrial low membrane potential cells, and the degreed of mitochondrial membrane permeability was enhanced. These suggested that Cu could cause mitochondrial dysfunction and structural impairment in kidney.

In this study, the mitochondrial dynamics was evaluated. Mitochondrial dynamics is a process that can be defined as spatiotemporal changes in mitochondrial structure, number and location within the cell. Fission and fusion in mitochondria are required for homeostasis and mitochondrial health especially when cells suffer from various stimuli and conditions [19]. Mitochondrial fission is a process in which daughter mitochondria are produced from the mother mitochondria leading to improve mitochondrial network fission. In addition, the separation of dysfunctional mitochondrial section requires Drp1 to be recruited into $\mathrm{OMM}$ leading to membrane depolarization. However, excess fission within mitochondria can impair metabolic function and derange mitochondrial dynamics [37]. In contrast to fission, mitochondrial fusion is a collaborative process that two originally different mitochondria physically merge into one. It also includes the fusion of $\mathrm{OMM}$ and the inner mitochondrial membrane (IMM). The fusion of OMM is regulated by Mfn1 and Mfn2, and fusion of the IMM is controlled by OPA1 [38]. In the present study, the mRNA and protein levels of Drp1 were increased markedly with the increased contents of $\mathrm{Cu}$. However, the mRNA and protein levels of mitochondrial fusion-related protein including OPA1, Mfn1, and Mfn2 were decreased significantly with the Cu contents elevated. These suggest that excess $\mathrm{Cu}$ could cause the excessive mitochondrial fission, which leading to mitochondrial homeostasis disequilibrium.

Mitochondria can be considered as a key factor in programmed cell death, and many studies have found that the mitochondrial-mediated apoptosis can be induced by types of toxic factors [39, 40]. Upon most occasions, mitochondria-mediated apoptosis is controlled by Bcl-2 family which consists of both proapoptotic factors (Bax/Bak) and anti-apoptotic factor Bcl-2. Once apoptotic signals are received, the proapoptotic proteins will transfer to the mitochondrial membrane and $\mathrm{Bcl}-2$ protein will down-regulated, 
which lead to permeabilize the OMM, MMP decreasing, and release of intermembrane pro-apoptotic substances, including CytC. And then, Caspase-3 stops contact with peripheral cells, reassembles the cytoskeleton, discontinues DNA duplication, destroys DNA, disrupts the nuclear structure, and disintegrates the cells into apoptotic bodies [14]. Additionally, p53 can exert a crucial part in regulating cell-cycle arrest, DNA double-stand break and mitochondria-dependent apoptosis in response to various stresses [41]. In present study, the decrease of MMP was accompanied by an increase of MPTP in kidney induced by $\mathrm{Cu}$. Furthermore, the mRNA and protein expression levels of Bax, Bak-1, CytC, and cleaved Caspase- 3 and the mRNA expression level of p53 were enhanced with the increased Cu level, but the mRNA and protein levels of Bcl-2 were down-regulated. This finding was further confirmed that excessive intake of Cu could cause mitochondria-mediated apoptosis in kidney.

\section{Conclusion}

In this study, metabolomics was used for the first time to evaluate mitochondria-mediated apoptosis caused by $\mathrm{Cu}$ in kidney. The mechanisms of Cu-induced apoptosis involve the metabolic pathways including riboflavin metabolism, glutathione metabolism, sphingolipid metabolism, and glycerophospholipid metabolism. Furthermore, excess $\mathrm{Cu}$ could induce mitochondria-mediated apoptosis in kidney, which might be closely related to above metabolic pathways. Thus, metabolomics, the most closely reflecting the phenotype, was effective in investigating the toxic mechanisms induced by $\mathrm{Cu}$ exposure in kidney of broiler chickens.

\section{Abbreviations}

CCCP: Carbonyl cyanide m-chlorophenylhydrazone

Cu: Copper

CytC: Cytochrome C

DAB: diaminobenzidine

Drp1: Dynamin-related protein 1

H\&E: Hematoxylin eosin

IMM: Inner mitochondrial membrane

LC-MS: Liquid chromatography-mass spectrometry

MAX: Maximal respiration

MMP: Mitochondrial membrane potential

MPTP: Mitochondria permeability transition pore 
OCR: Oxygen consumption rate

OMM: Outer mitochondrial membrane

OPLS-DA: Orthogonal partial least squared discriminant

PCA: Principle component analysis

PBS: Phosphate buffer solution

RCR: Respiratory control ratio

ROS: Reactive oxygen species

SRC: Spare respiratory capacity

VIP: variable importance in the projection

\section{Declarations}

\section{Ethics approval and consent to participate}

The procedures were authorized by the Ethics Committee of South China Agricultural University (Permission number: NO. 2017A087).

\section{Consent for publication}

Not applicable.

\section{Availability of data and material}

All the data generated and analyzed during the current study were included in this study.

\section{Competing interests}

No conflict of interest.

\section{Funding}

This research was funded by the National Natural Science Foundation of China (No. 32072930 and No. 31572585) and the National Key R \& D Program of China (2016YFD0501205 and No. 2017YFD0502200). 


\section{Authors' contributions}

Z.T., J. L., and F. Y. designed the study; J. L. and F. Y. wrote the paper; J. L., Y. B., W. Y., and N. Q. analyzed data; Q. H., H. Z., J. G., L. H., Y. L., and J. P. revised the paper. The authors approved the final manuscript.

\section{Acknowledgements}

Not applicable.

\section{References}

1. Zhao J, Shirley RB, Dibner JJ, Wedekind KJ, Yan F, Fisher P, Hampton TR, Evans JL, Vazquez-Anon M. Superior growth performance in broiler chicks fed chelated compared to inorganic zinc in presence of elevated dietary copper. J Anim Sci Biotechnol. 2016;7:13.

2. Olukosi OA, van Kuijk S, Han Y. Copper and zinc sources and levels of zinc inclusion influence growth performance, tissue trace mineral content, and carcass yield of broiler chickens. Poultry Sci. 2018;97:3891-3898.

3. Liao J, Yang F, Chen H, Yu W, Han Q, Li Y, Hu L, Guo J, Pan J, Liang Z, Tang Z. Effects of copper on oxidative stress and autophagy in hypothalamus of broilers. Ecotoxicol Environ Saf. 2019;185:109710.

4. Nastasescu V, Mititelu M, Goumenou M, Docea AO, Renieri E, Udeanu DI, Oprea E, Arsene AL, DinuPirvu CE, Ghica M. Heavy metal and pesticide levels in dairy products: Evaluation of human health risk. Food Chem Toxicol. 2020;146:111844.

5. Espinosa CD, Stein HH. Digestibility and metabolism of copper in diets for pigs and influence of dietary copper on growth performance, intestinal health, and overall immune status: a review. J Anim Sci Biotechnol. 2021;12:13.

6. Yang F, Cao H, Su R, Guo J, Li C, Pan J, Tang Z. Liver mitochondrial dysfunction and electron transport chain defect induced by high dietary copper in broilers. Poultry Sci. 2017;96:3298-3304.

7. Jomova K, Lawson M, Drostinova L, Lauro P, Poprac P, Brezova V, Michalik M, Lukes V, Valko M. Protective role of quercetin against copper(II)-induced oxidative stress: A spectroscopic, theoretical and DNA damage study. Food Chem Toxicol. 2017;110:340-350.

8. Yang F, Liao J, Pei R, Yu W, Han Q, Li Y, Guo J, Hu L, Pan J, Tang Z. Autophagy attenuates copperinduced mitochondrial dysfunction by regulating oxidative stress in chicken hepatocytes. Chemosphere. 2018;204:36-43.

9. Thomas VG, McGill IR. Dissolution of copper, tin, and iron from sintered tungsten-bronze spheres in a simulated avian gizzard, and an assessment of their potential toxicity to birds. Sci Total Environ. 2008;394:283-289. 
10. Wang Y, Zhao H, Yang X, Mu M, Zong H, Luo L, Xing M. Excessive Cu2+ deteriorates arsenite-induced apoptosis in chicken brain and resulting in immunosuppression, not in homeostasis. Chemosphere. 2020;239:124758.

11. Wan F, Zhong G, Ning Z, Liao J, Yu W, Wang C, Han Q, Li Y, Pan J, Tang Z, Huang R, Hu L. Long-term exposure to copper induces autophagy and apoptosis through oxidative stress in rat kidneys. Ecotoxicol Environ Saf. 2020;190:110158.

12. Zhao H, Wang Y, Fei D, Guo M, Yang X, Mu M, Yu H, Xing M. Destruction of redox and mitochondrial dynamics co-contributes to programmed cell death in chicken kidney under arsenite or/and copper (II) exposure. Ecotoxicol Environ Saf. 2019;179:167-174.

13. Nowak KL, Edelstein CL. Apoptosis and autophagy in polycystic kidney disease (PKD). Cell Signal. 2020;68:109518.

14. Abate M, Festa A, Falco M, Lombardi A, Luce A, Grimaldi A, Zappavigna S, Sperlongano P, Irace C, Caraglia M, Misso G. Mitochondria as playmakers of apoptosis, autophagy and senescence. Semin Cell Dev Biol. 2020a;98:139-153.

15. Rekha KR, Selvakumar GP. Gene expression regulation of $\mathrm{Bcl} 2$, Bax and cytochrome-C by geraniol on chronic MPTP/probenecid induced C57BL/6 mice model of Parkinson's disease. Chem-Biol Interact. 2014;217:57-66.

16. Abate M, Festa A, Falco M, Lombardi A, Luce A, Grimaldi A, Zappavigna S, Sperlongano P, Irace C, Caraglia M, Misso G. Mitochondria as playmakers of apoptosis, autophagy and senescence. Semin Cell Dev Biol. 2020b;98:139-153.

17. Sheridan C, Martin SJ. Mitochondrial fission/fusion dynamics and apoptosis. Mitochondrion 2010;10:640-648.

18. Dietz JV, Bohovych I, Viana MP, Khalimonchuk O. Proteolytic regulation of mitochondrial dynamics. Mitochondrion. 2019;49:289-304.

19. Khan S, Raj D, Jaiswal K, Lahiri A. Modulation of host mitochondrial dynamics during bacterial infection. Mitochondrion. 2020;53:140-149.

20. Cui G, Li Y, Ding K, Hao S, Wang J, Zhang Z. Attribution of Bax and mitochondrial permeability transition pore on cantharidin-induced apoptosis of Sf9 cells. Pestic Biochem Phys. 2017;142:91101.

21. Wishart DS. NMR metabolomics: A look ahead. J Magn Reson. 2019;306:155-161.

22. Mansano AS, Souza JP, Cancino-Bernardi J, Venturini FP, Marangoni VS, Zucolotto V. Toxicity of copper oxide nanoparticles to Neotropical species Ceriodaphnia silvestrii and Hyphessobrycon eques. Environ Pollut. 2018;243:723-733.

23. Oto J, Fernández-Pardo Á, Roca M, Plana E, Solmoirago MJ, Sánchez-González JV, Vera-Donoso CD, Martínez-Sarmiento M, España F, Navarro S, Medina P. Urine metabolomic analysis in clear cell and papillary renal cell carcinoma: A pilot study. J Proteomics. 2020;218:103723.

24. Yang YX, Yu S, Jia BX, Liu N, Wu A. Metabolomic profiling reveals similar cytotoxic effects and protective functions of quercetin during deoxynivalenol- and 15-acetyl deoxynivalenol-induced cell 
apoptosis. Toxicol In Vitro. 2020;66:104838.

25. Ledoux DR, Henry PR, Ammerman CB, Rao PV, Miles RD. Estimation of the relative bioavailability of inorganic copper sources for chicks using tissue uptake of copper. J Anim Sci. 1991;69(1):215-222.

26. Zhang C, Qin L, Dou DC, Li XN, Ge J, Li JL. Atrazine induced oxidative stress and mitochondrial dysfunction in quail (Coturnix C. coturnix) kidney via modulating Nrf2 signaling pathway. Chemosphere. 2018;212:974-982.

27. Zhang C, Li H, Qin L, Ge J, Qi Z, Talukder M, Li YH, Li JL. Nuclear receptor AHR-mediated xenobiotic detoxification pathway involves in atrazine-induced nephrotoxicity in quail (Coturnix C. coturnix). Environ Pollut. 2019;253:889-898.

28. Luan Y, Li Y, Yue X, Cao Y, Xiang F, Mao D, Xiong Z. Metabonomics of mice intestine in Codonopsis foetens induced apoptosis of intestine cancer cells. SAUDI J Biol Sci. 2019;26:1003-1010.

29. Jang DH, Shofer FS, Weiss SL, Becker LB. Impairment of mitochondrial respiration following ex vivo cyanide exposure in peripheral blood mononuclear cells. Clin Toxicol (Phila). 2016;54:303-307.

30. Garcia-Roche M, Casal A, Carriquiry M, Radi R, Quijano C, Cassina A. Respiratory analysis of coupled mitochondria in cryopreserved liver biopsies. Redox Biol. 2018;17:207-212.

31. Lee YS, Kang YS, Lee SH, Kim JA. Role of NAD(P)H oxidase in the tamoxifen-induced generation of reactive oxygen species and apoptosis in HepG2 human hepatoblastoma cells. Cell Death Differ. 2000;7:925-932.

32. Li N, Ragheb K, Lawler G, Sturgis J, Rajwa B, Melendez JA, Robinson JP. DPI induces mitochondrial superoxide-mediated apoptosis. Free Radic Biol Med. 2003;34:465-477.

33. Zhu Y, Wu X, Liu Y, Zhang J, Lin D. Integration of transcriptomics and metabolomics reveals the responses of earthworms to the long-term exposure of TiO2 nanoparticles in soil. Sci Total Environ. 2020;719:137492.

34. Botvinik LA, Al QA, Yekhtin Z, Srebnik M, Dagan A. Novel 3-hydroxy vinylboronates influence sphingolipid metabolism, cause apoptosis in Jurkat cells and prevent tumor development in nude mice. Bioorg Med Chem Lett. 2013;23:507-512.

35. Lu Z, Li Y, Ru JH, Lopes-Virella MF, Lyons TJ, Huang Y. Interaction of palmitate and LPS regulates cytokine expression and apoptosis through sphingolipids in human retinal microvascular endothelial cells. Exp Eye Res. 2019;178:61-71.

36. Gonzalez-Baro MR, Coleman R.A. Mitochondrial acyltransferases and glycerophospholipid metabolism. BBA-Mol Cell Biol L. 2017;1862:49-55.

37. Seo BJ, Choi J, La H, Habib O, Choi Y, Hong K, Do JT. Role of mitochondrial fission-related genes in mitochondrial morphology and energy metabolism in mouse embryonic stem cells. Redox Biol. 2020;36:101599.

38. Wang H, Liu J, Wei S, Zhao W, Zhu S, Zhou B. Mitochondrial respiratory chain damage and mitochondrial fusion disorder are involved in liver dysfunction of fluoride-induced mice. Chemosphere. 2020;241:125099. 
39. P V, D D, Bala M, N S. Photoactivated [Mn(CO)3Br( $\mu$-bpcpd)]2 induces apoptosis in cancer cells via intrinsic pathway. J Photoch Photobio B. 2018;188:28-41.

40. Li Z, Li Q, Lv W, Jiang L, Geng C, Yao X, Shi X, Liu Y, Cao J. The interaction of Atg4B and Bcl-2 plays an important role in $\mathrm{Cd}$-induced crosstalk between apoptosis and autophagy through disassociation of Bcl-2-Beclin1 in A549 cells. Free Radic Biol Med. 2019;130:576-591.

41. Shi K, An J, Qian K, Zhao X, Li F, Ma X, Wang Y, Zhang Y. p53 controls the switch between autophagy and apoptosis through regulation of PLSCR1 in sodium selenite-treated leukemia cells. Exp Cell Res. 2020;389:111879.

\section{Figures}
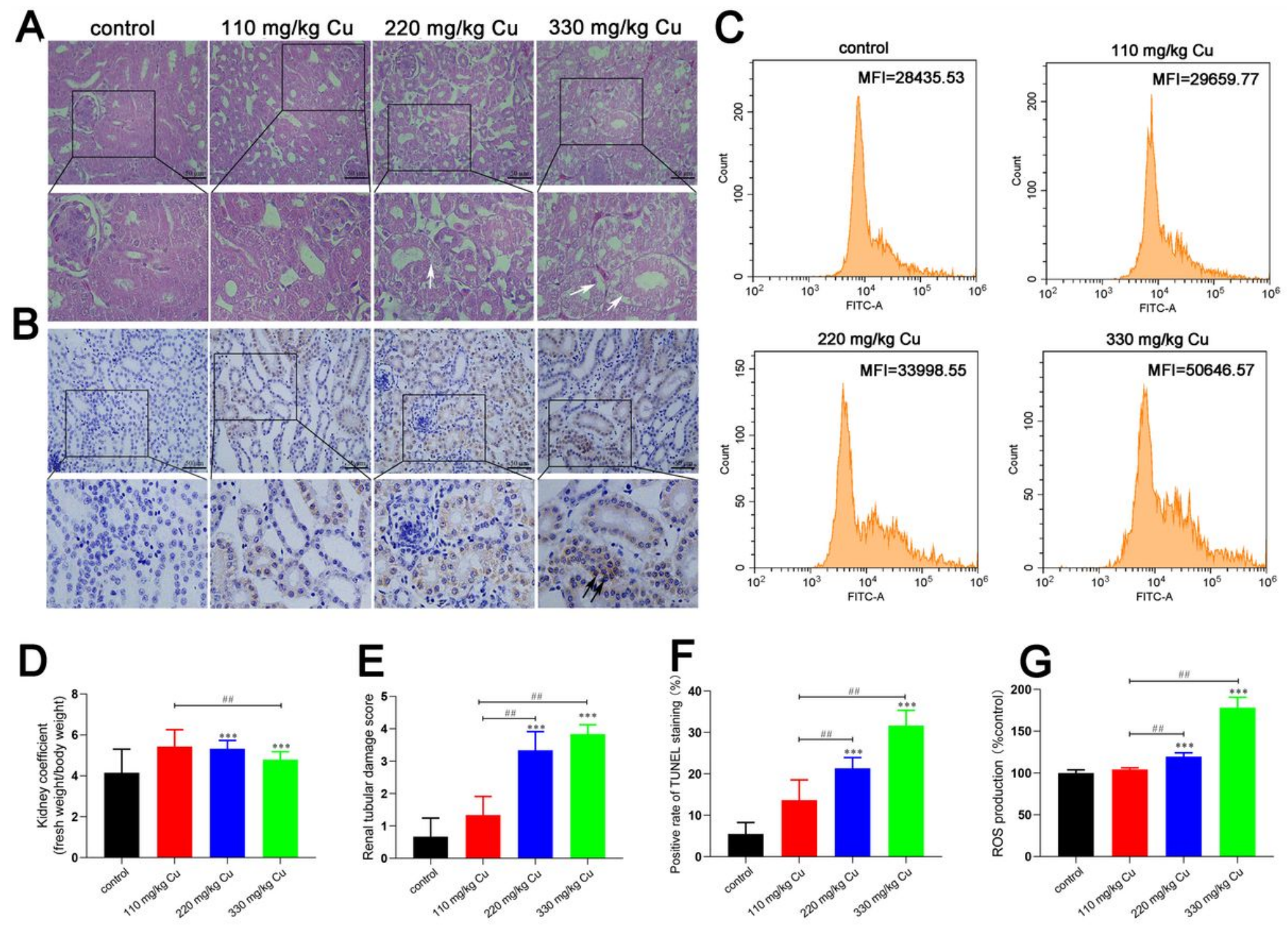

Figure 1

Effects of dietary Cu on histological morphology, TUNEL staining, and ROS production in kidney. (A) Histological morphology. The plotting scale was $50 \mu \mathrm{m}$. White arrows indicated vacuolar degeneration. (B) TUNEL staining. The plotting scale was $50 \mu \mathrm{m}$. Black double arrows indicated TUNEL-positive cells. 
(C) ROS levels were detected by flow cytometry. (D) Kidney coefficient. (E) Renal tubular damage score.

(F) Positive rate of TUNEL staining. (G) Quantitative analysis of ROS levels. Data were presented as mean $\pm S E(n=6)$. "*” represented statistical difference compared to control group $\left({ }^{*} P<0.05,{ }^{* *} P<0.01\right.$ or $\star \star \star P<0.001)$. "\#" represented statistical difference between two groups $(\# \mathrm{P}<0.05, \# \# \mathrm{P}<0.01$ or $\# \# \# \mathrm{P}<0.001)$, similarly hereinafter.

A

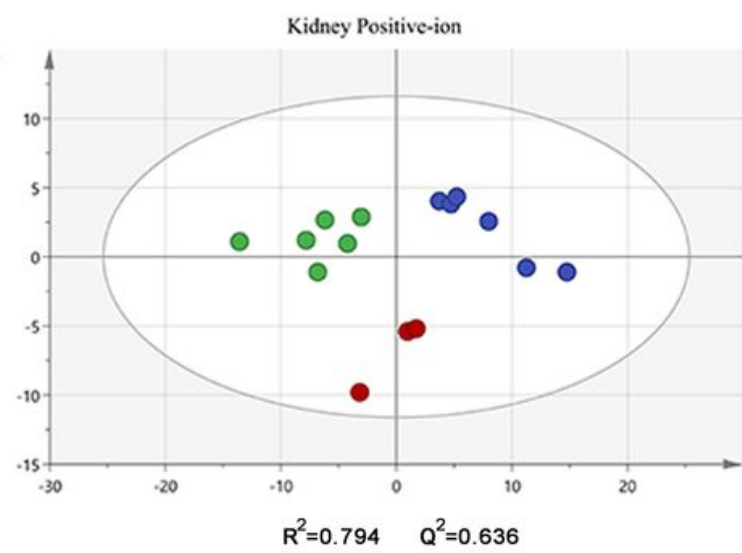

C

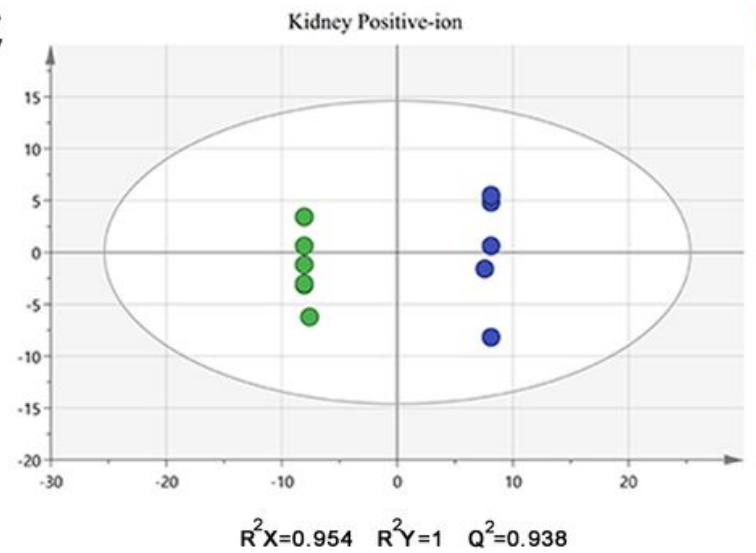

E

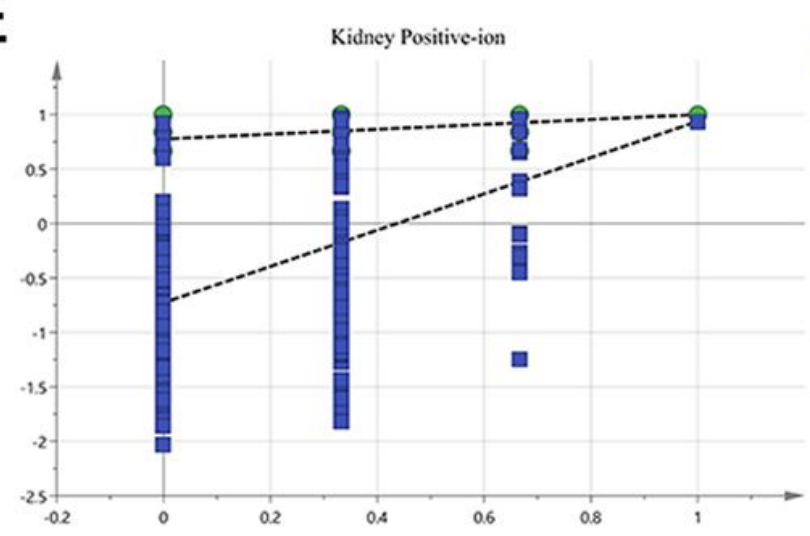

control $330 \mathrm{mg} / \mathrm{kg} \mathrm{Cu}$

$330 \mathrm{~m}$
$\mathrm{QC}$

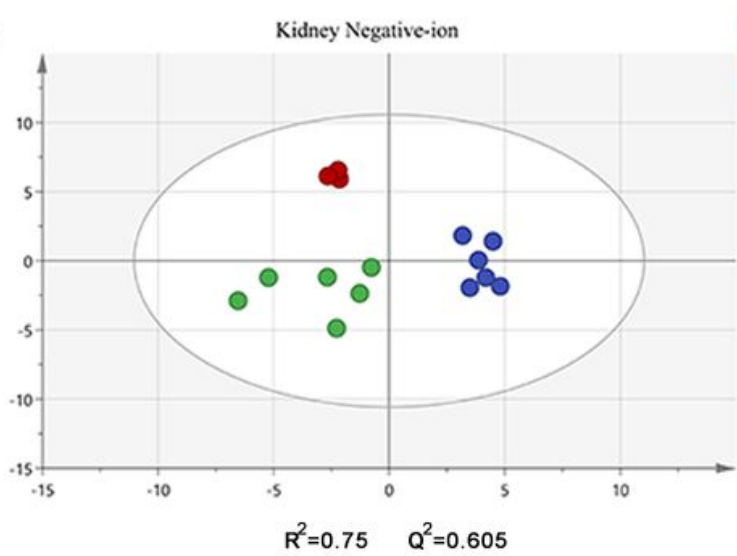

control
$330 \mathrm{mg} / \mathrm{kg} \mathrm{Cu}$
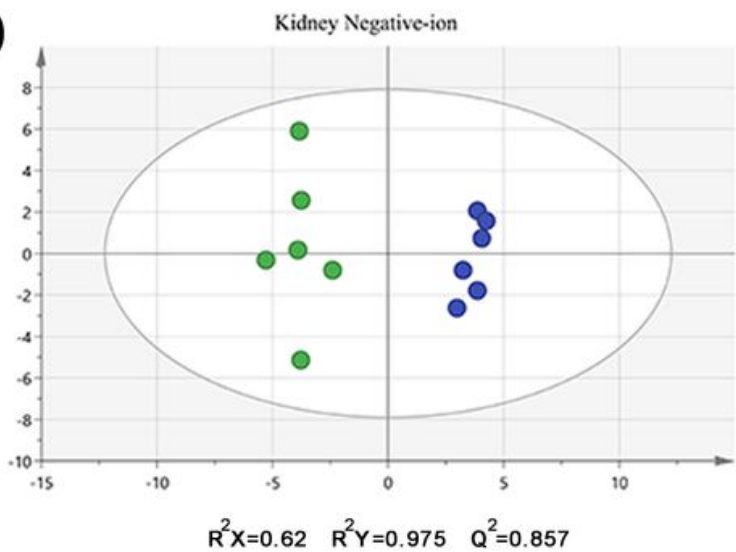

$\underset{\substack{\mathrm{R}^{2} \\ \mathrm{a}^{2}}}{\mathrm{~F}}$

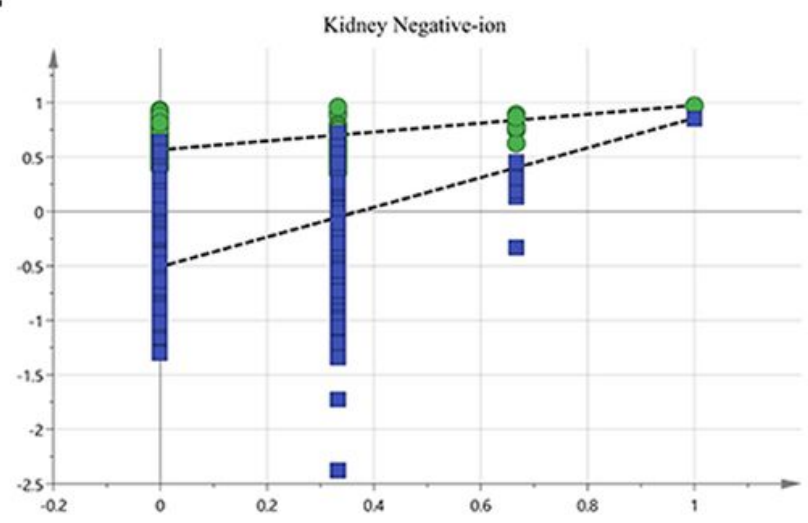

Figure 2

Multivariate statistical analysis of metabolites in kidney. (A-B) PCA score plots of the control group and $330 \mathrm{mg} / \mathrm{kg}$ Cu group in positive-ion mode (A) and negative-ion mode (B). (C-D) OPLS-DA plot of the control group and $330 \mathrm{mg} / \mathrm{kg}$ Cu group in positive-ion mode (C) and negative-ion mode (D). (E) 
Permutation tests of the OPLS-DA mode (positive-ion). (F) Permutation tests of the OPLS-DA mode (negative-ion).
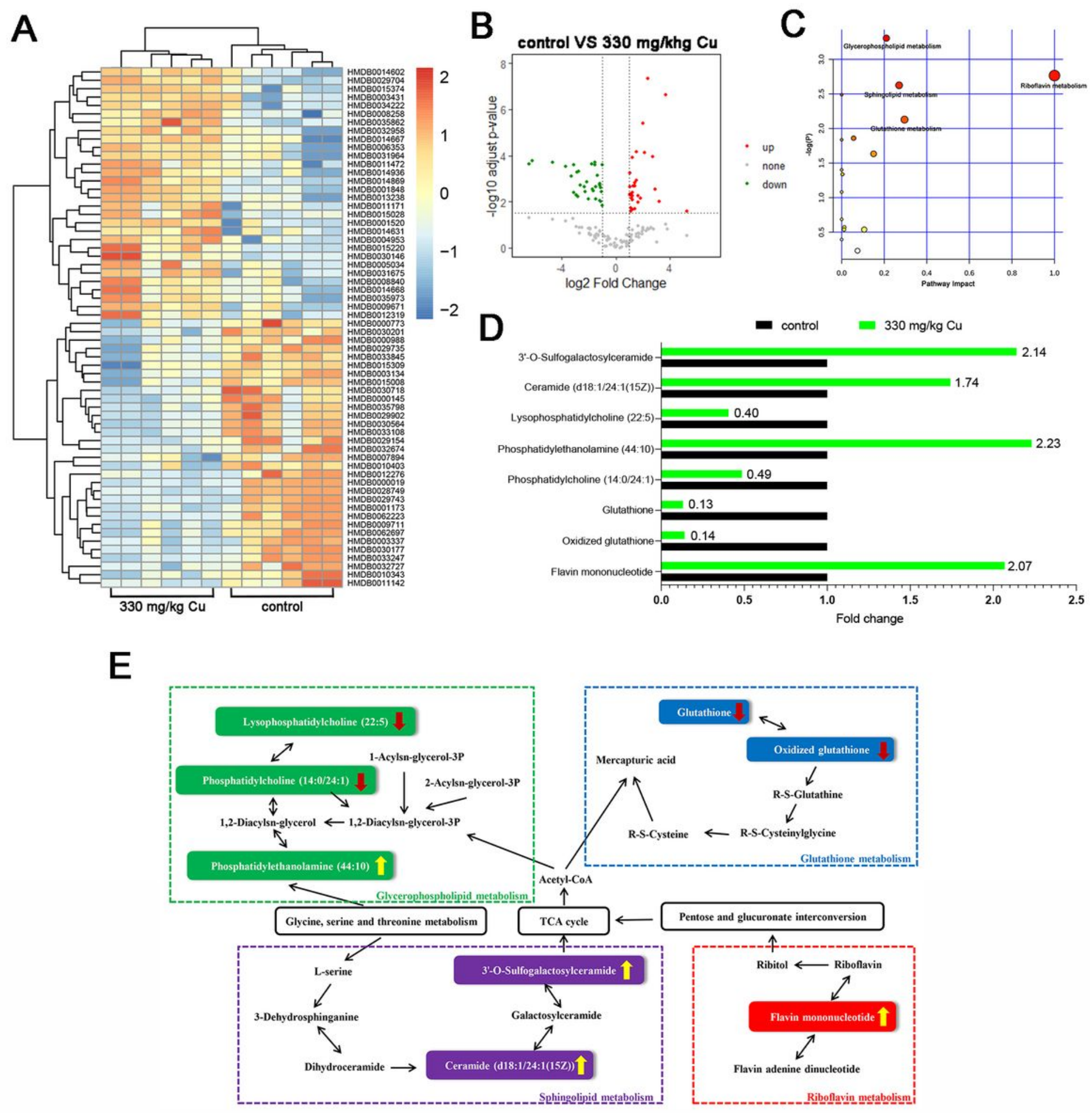

\section{Figure 3}

The nephrotoxicity of Cu was analyzed by metabolomics. (A) Differential metabolites were exhibited in heatmap. (B) Differential metabolites were exhibited in volcano plots. (C) Pathway analysis of metabolites in kidney of broiler chickens. (D) Different metabolites related to riboflavin metabolism, 
glutathione metabolism, sphingolipid metabolism, and glycerophospholipid metabolism. (E) Metabolic diagram in kidney exposed to $330 \mathrm{mg} / \mathrm{kg}$ Cu compared to control group. The metabolites in green boxes were involved in glycerophospholipid metabolism. The metabolites in blue boxes were involved in glutathione metabolism. The metabolites in purple boxes were involved in sphingolipid metabolism. The metabolites in red boxes were involved in riboflavin metabolism. The yellow arrow meant up-regulated in $330 \mathrm{mg} / \mathrm{kg}$ Cu group compared to control group, and the red arrow meant down-regulated.
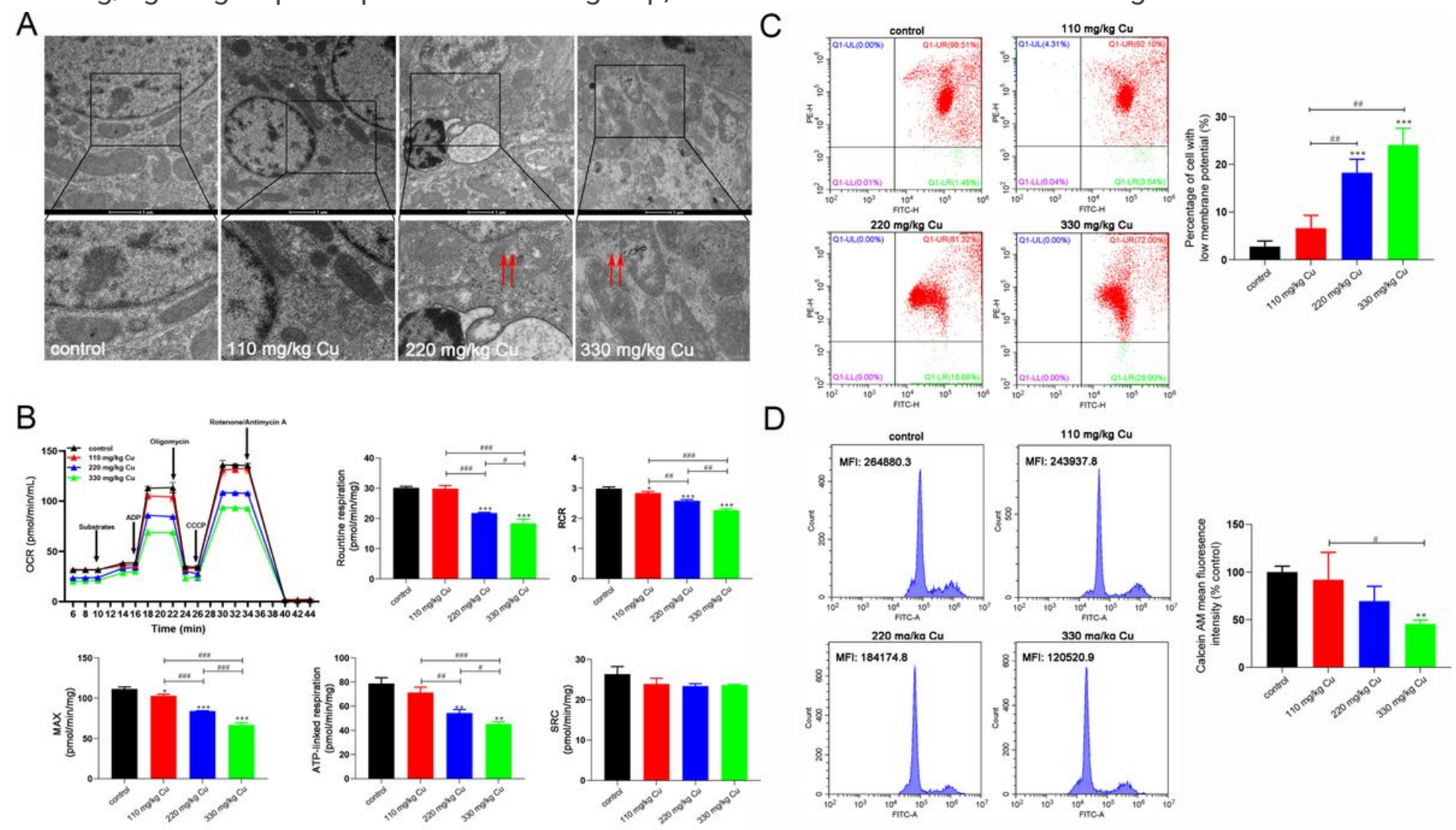

Figure 4

The evaluation of mitochondrial damage in kidney. (A) Ultrastructure. The plotting scale was $1 \mu \mathrm{m}$. The red double arrows indicated damaged mitochondria. " $\mathrm{N}$ " meant nucleus, and "mito" meant mitochondria. (B) Mitochondrial respiratory function. (C-D) The decreased of MMP and increased of MPTP induced by $\mathrm{Cu}$ in kidney. Flow cytometry was used to detect MMP with JC-1 (C) and MPTP with Calcein AM (D). 
A
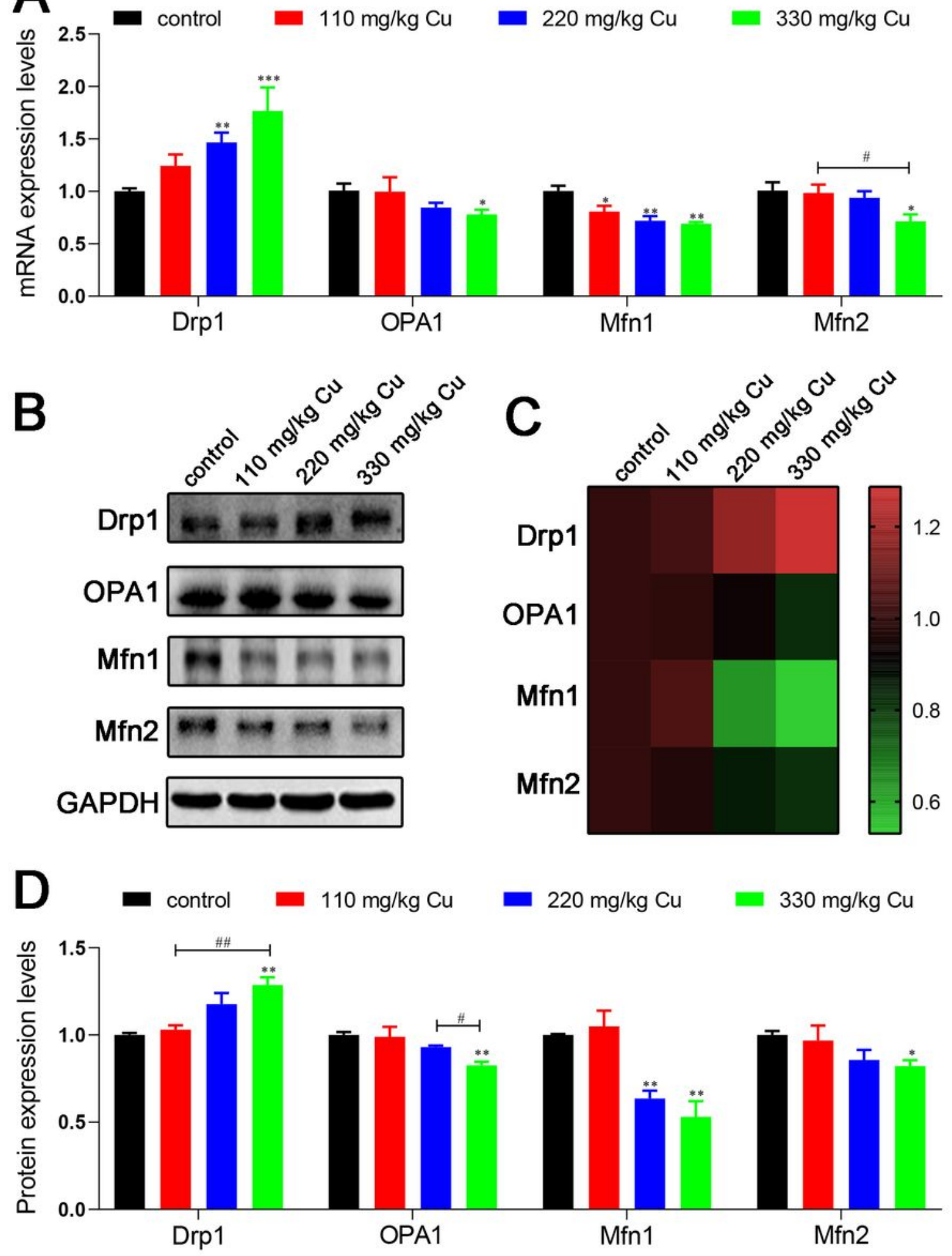

Figure 5

The mRNA and protein levels of mitochondrial dynamics protein in kidney. (A) mRNA expression of mitochondrial dynamics protein. (B) Western blot showed that expression of mitochondrial dynamics protein. (C) Heatmap showed the protein expression of mitochondrial dynamics protein. (D) Histogram showed the protein levels of mitochondrial dynamics protein. 

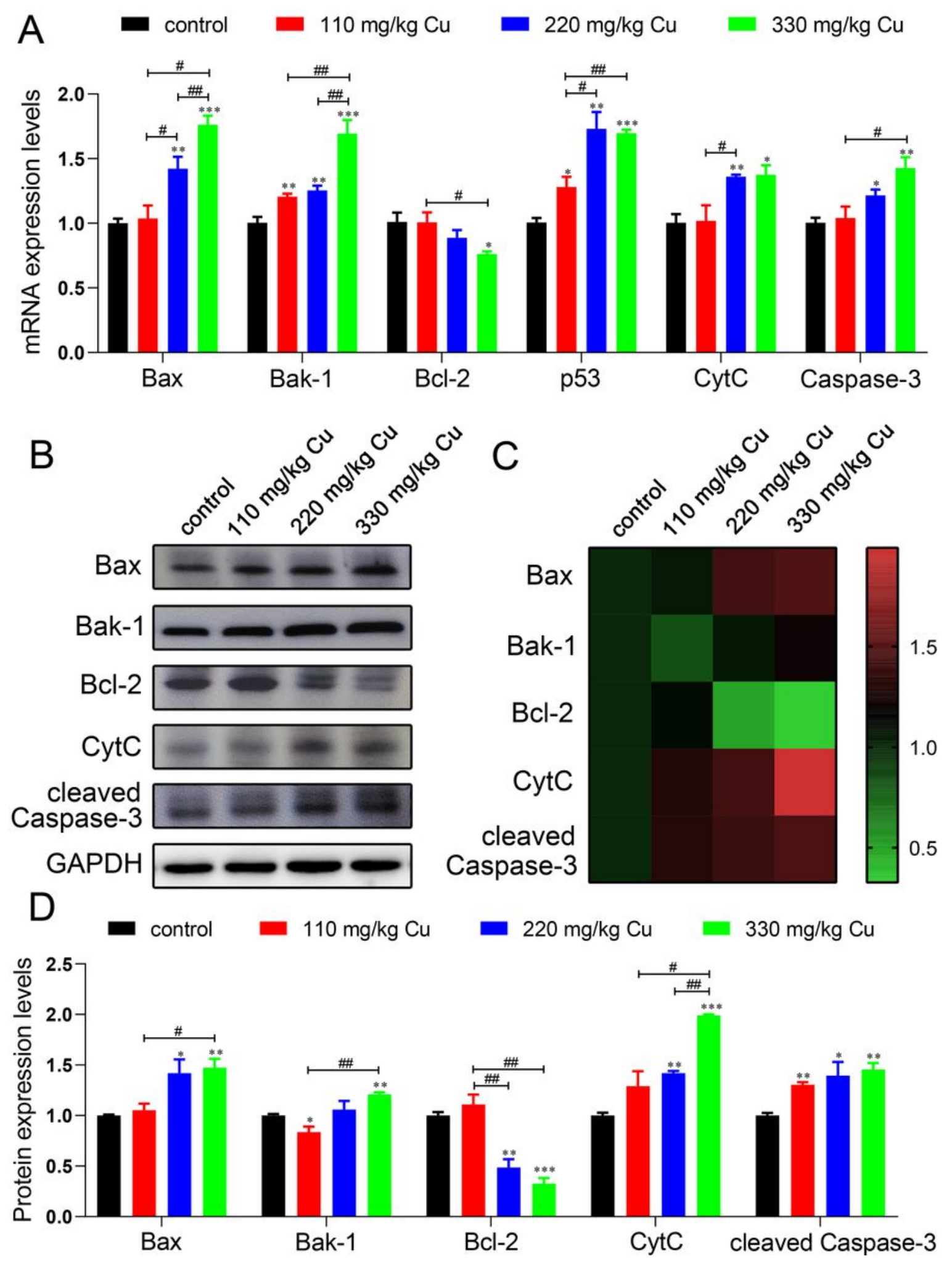

Figure 6

The expressions of mitochondria-dependent apoptosis-related genes and proteins induced by $\mathrm{Cu}$ in kidney. (A) mRNA expression of mitochondria-dependent apoptosis-related genes. (B) Protein bands of mitochondrial-dependent apoptosis-related protein. (C) Heatmap showed the expression of mitochondriamediated apoptosis-related protein. (D) Histogram showed the protein expressions of mitochondriadependent apoptosis-related protein. 


\section{Supplementary Files}

This is a list of supplementary files associated with this preprint. Click to download.

- supplementarymaterial.doc 